RECENT exciting advances in physics at millikelvin temperatures have been made possible by an ample and relatively cheap supply of helium-3 for operating dilution refrigerators, but it looks very much as though this happy situation is now coming to an end, with an impending world-wide shortage of the gas. The Radiochemical Centre at Amersham, for example, is not accepting any further orders: even when the present backlog of orders, some dating back to the beginning of the year, has finally been cleared, it is apparently by no means certain that further supplies will become available for sale.

One of the principal uses of helium3 is as the working fluid of the dilution refrigerators which have become the work-horses of millikelvin research. The principle on which they operate is extremely simple, just depending on the cooling effect which takes place when liquid helium-3 is allowed to dissolve in liquid helium-4. The refrigerator is usually arranged so that the process is continuous, with provision being made for separating the two isotopes again in a still, and for recirculating the ${ }^{3} \mathrm{He}$ back to the mixing chamber through a series of heat exchangers in which it is cooled by the cold dilute mixture leaving the chamber. Optimising the design of such machines is quite a complicated exercise, but progress over the past few years has been rapid: the refrigerators developed in Grenoble by Frossati, for example, are now able to sustain temperatures of around $2.5 \mathrm{mK}$ in continuous operation, which is lower by a factor of four than the best available commercial machines of only a couple of years ago.

The present helium-3 famine arises, probably, for three main reasons. First, there has been a decided increase in the number of research

\section{Will detente kill millikelvin research?}

from P. V. E. McClintock

groups building or buying dilution refrigerators, partly stimulated by the discovery of superfluidity in liquid helium-3 itself: all these machines require an initial charge of helium-3. Second, the relatively large machines which people are now tending to build require correspondingly large quantities of helium-3; this is particularly true of the Frossati designs, which incorporate exceptionally large diameter tubes within and between the lower heat-exchangers in order to reduce viscous heating effects as the liquid moves through the system. Finally, there is detente.

The ample supply of helium-3 enjoyed by cryophysicists in recent years has been produced as a byproduct of the nuclear weapons programme. The tritium used in thermonuclear devices decays through $\beta$-emission to helium3 , with a half-life of about 12 years. Although nobody seems very keen on discussing the exact arrangements made in practice for collecting the helium-3, one may infer that it is very much less convenient to do so once the weapon is in its final form ready for use, so that the ready availability of gas for sale to researchers and the cryogenics industry probably depends on continued manufacture.

Although the dilution refrigerator in its present form can certainly be regarded as a byproduct of the thermonuclear arms race, and any slackening of the latter must be warmly welcomed, it will be sad indeed if the excitingly rapid progress now being made in the millikelvin field is seriously curtailed. There are, however, two reasons to remain fairly optimistic about the long term outcome.

First, one may note that after being set up with its initial charge of helium-3, very little of the gas need get lost from a dilution refrigerator, such losses as there are occurring only by leakage or through human error, both of which can be minimised by careful design. So, provided that there is a slowdown in the rate at which new machines are being commissioned, the present gap between demand and supply may eventually decrease again. Unfortunately there is no sign of this happening just at the moment.

Second, there always remains the possibility of extraction from natural helium-4, which usually contains a few parts in $10^{7}$ of the light isotope. This certainly can be accomplishedat a price-and, indeed, was used as a small scale method of helium-3 production before the advent of extensive thermonuclear weapons programmes. There is a considerable variation in the helium- 3 content of the gas from different wells. By choosing the right well and using modern isotopic separation techniques it just might be possible to produce helium-3 at a price not too much above the present $£ 75$ per S.T.P. litre. Furthermore, if the rumours about certain Polish gas wells prove to be correct-that they produce helium containing about 1 part in $10^{5}$ of helium-3-a return to extraction from the natural helium could even lead to a reduction in the current price, at the same time making Poland the World's chief supplier of helium-3.

Until an alternative source of helium-3 is developed, detente is undoubtedly going to bring a measure of deprivation to the millikelvinists.

P. V. E. McClintock is in the Department of Physics, University of Lancaster. membranes is likely to provide a novel approach to the exploration of membrane function.

\section{Complexity and stability}

from N. MacDonald

THE relationship between the complexity and the stability of an ecosystem has been debated for some years by mathematically inclined ecologists, and has occasioned the choice of title for an extremely influential book (May, Stability and Complexity in Model Ecosystems, Princeton, 1973). A possible relation- ship is indicated by a simple argument concerning a food chain, in which species A depends solely on species B, species B solely on species $C$, and so on. Any break in the chain eliminates all the previous members A,B ... On the other hand, if there are alternative paths through a food network, one may expect the general structure of the network to survive the loss of one species. Here complexity is interpreted as the fraction of the pairs of species which directly interact, a quantity which may be termed connectance. Stability is interpreted as survival of all remaining

N. MacDonald is in the Department of Natural Philosophy, University of Glasgow. species when one is lost.

As one makes the model more mathematically specific, so one has to be specific in the choice of a measure of complexity, and in the type of stability property that is studied. Some version of connectance, of ten allowing for an average over interactions of different strengths rather than simply counting the interactions, is usually the measure of complexity, in particular in a series of papers which appeared in Nature a few years ago. (Gardiner \& Ashby, Nature 228, 784; 1971; May, ibid 238, 413; 1972; Siljak, ibid 249, 280; 1974; Daniels \& Mackay, ibid 251, 49; 1974; Roberts, ibid 251, 607; 1974; Gilpin, ibid 254, 137; 1975; Saunders \& Bazin, ibid 256, 